\title{
A Genetic Machine Learning Algorithm for Load Balancing in Cluster Configurations
}

\author{
M.A.R. Dantas and A.R. Pinto \\ Department of Informatics and Statistics, \\ Federal University of Santa Catarina, \\ 88040-900 Florianopolis Brazil \\ \{mario, arpinto\}@inf.ufsc.br
}

\begin{abstract}
Cluster configurations are a cost effective scenarios which are becoming common options to enhance several classes of applications in many organizations. In this article, we present a research work to enhance the load balancing, on dedicated and non-dedicated cluster configurations, based on a genetic machine learning algorithm. Classifier systems are learning machine algorithms, based on high adaptable genetic algorithms. We developed a software package which was designed to test the proposed scheme in a master-slave Cow and Now environment. Experimental results, from two different operating systems, indicate the enhanced capability of our load balancing approach to adapt in cluster configurations.
\end{abstract}

\section{Introduction}

Dedicated and non-dedicated cluster configurations offer a cost/effective highperformance computing if idle resources from these clusters can be successful harnessed. Several package environments (such as OSCAR (Open Source Cluster Application Resource)[1] and Linux Virtual Server [2]) were developed to provide functions of a single system image. These environments improve the use of cluster architectures, providing many important mechanisms to help application programmers to achieve a high performance. The load balancing issues in distributed systems (such as cluster environments) have been studied by a number of researches and many points have already been addressed $[3,4,5]$.

However, a well known problem in implementing software environments for single system image is the use of an effective technique to distribute processes to processors in a fair based policy [6]. As many research works have already demonstrated (e.g. $[5,6]$ ), in a cluster of computers it is high the possibility of a specific node become heavily loaded and others lightweight loaded.

The load balancing problem is recognized as a NP-complete problem [7]. Therefore, application developers commonly use heuristic or stochastic methods because those two paradigms can obtain sub-optional results in a reasonable period of time. In the literature we can observe an expressive utilization of genetic algorithm. 
In this article, we present an approach to schedule processes using a classifier system. This method has an interesting feature of machine learning [8] based on a genetic algorithm. The results were obtained considering two configurations. The first configuration was a cluster of tightly machines (Cow) using Linux with the OSCAR single system image. The other environment was an ordinary network of workstations (Now) with loosely coupled machines executing the Windows operating system. The proposed system was implemented in Java.

The paper is organized as follows. Section 2 presents some concepts of scheduling and load balancing approaches. Concepts of genetic algorithms and classifier systems are shown in section 3. Our proposal is described in section 4. Experimental results of a parallel application are presented in section 5. Finally, in section 6 we draw some conclusions about the present research and our contribution, indicating some future directions for this research work.

\section{Scheduling and Load Balancing in Parallel and Distributed Systems}

The taxonomy presented in [4], considers the global scheduling as static and dynamic. The static approach requires previous knowledge of processes behavior and its dependence. Therefore, the static technique is more suitable for homogeneous environments, because this method knows processes behavior at the compilation time. On the other hand, the dynamic scheduling is used when we do not know any previous requirements of processes and environments. Thus, in this paradigm we should implement queries which interact frequently with the configuration to gather information of the load of the processors. In addition, a strict dynamic method considers the migration of processes when a processor became overload for some reason. However, this technique is a complex task and has a high computational cost (e.g. [9]). Therefore, it is also possible to implement a more relaxed approach of dynamic scheduling migration called on time assignment scheduling (OTS).

\section{Genetic Algorithms and Classifier Systems}

Genetic algorithms are designed to perform a search, based on mechanisms of natural selection. In nature the most adapted individuals have more chances to survive. In addition, they provide their genetic code to their descendants. An individual in a genetic algorithm is represented by its chromosome, which is usually a string. In a new generation, new artificial creatures (strings) are created using fragments of the most adapted individuals from the old generation.

A classifier system is a genetic based machine learning algorithm, that can learn syntactically simple rules (called classifiers) to guide its performance in an arbitrary environment [8]. A classifier system has 3 main modules: rule and message system, a special class of production system; apportionment of credit algorithm; genetic algorithm. A production system is a computational scheme that 
employs rules as the main algorithmic goal. Rules usually present the following form:

If $<$ condition $>$ then $<$ action $>$

If the classifier system receives the message "101011" the first classifier will be activated and the action "100" will be executed. During the creation of a classifier system, all classifiers have the same fitness. When a classifier is chosen, it has to pay a certain amount of its fitness to the apportionment of credit algorithm. The amount of fitness is determined by a pre-defined tax. The paid amount is the product between the pre-defined tax and the classifier fitness. This amount will be paid in the next classifier system consultation, if the previous classifier sent an action that improves the system performance. Otherwise, the amount will be accumulated until a classifier gives a positive action. After a pre-defined number of consults, genetic algorithm is activated. The population of classifiers is replaced.

\section{Proposed Environment}

A large number of process scheduling researches that using genetic algorithm are based on the simulation of the environment. In contrast, we decided to design and implement a solution using real cluster configurations.

The first step in developing a global solution to schedule process, based on classifier systems, is to choose a distributed paradigm. In our case we decided to implement a master-slave approach. Our classifier system proposal is to develop a modified threshold method. This algorithm provides a process threshold indicating the number of processes which each can execute. We consider the average response time variation of processes as entry condition to the classifier. The configuration of classifiers will be: $<$ if a grow occurs or a decrement $>+<$ variation rate of average response time $>:<$ if the threshold should be incremented or decremented $>+<$ threshold rate variation $>$

\section{$5 \quad$ Experimental Results}

The performance of the classifier system was compared to random and threshold methods. In the random method a destination node is randomly chosen to receive processes. On the other hand, the threshold method pre-defines a maximum number of processes.

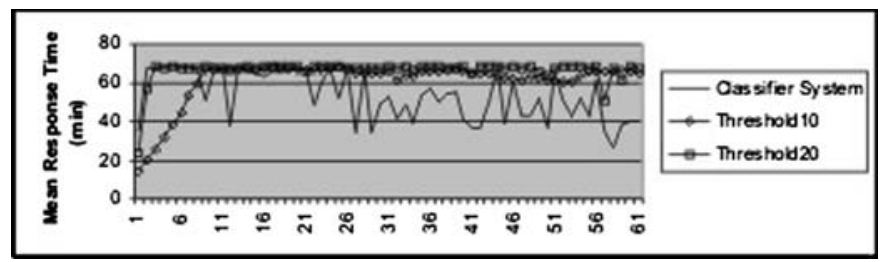

Fig. 1. Second experimental results 
The first experiment was executed in the non-dedicated environment. In the last experiment we consider an OSCAR cluster configuration. The processes submitted to system in frits experiment was a for loop to calculate a sum. On the other hand, the processes submitted to OSCAR configuration calculated the prime numbers by Eratostenes ciev. We consider the average response time metric, therefore a small average response time mean a better performance. The second experiment was idealized for test the robustness of our proposal. Therefore, we execute in the dedicated cluster for 20 hours, with interval of 20 minutes. As figure 1 shows the system had an interesting behavior. The proposed classifier system shows a high adaptability that was our primarily goal.

\section{Conclusion and Future Work}

The experimental results present in this article show the high adaptive characteristic of our proposed classifier system implementation. In addition, it demonstrates the capability of learning about cluster configurations (non-dedicated and dedicated environments). These facts are confirmed by intervals where we had low performance compared to the following enhanced performance. As future work we are planning a scheme to store classifiers, therefore when the system begins the execution classifiers would be adapted to the reality which they will execute.

\section{References}

1. Benoit Ligneris, Stephen Scott, Thomas Naughton, Neil Gorsuch, Open Source Cluster Application Resource (OSCAR): Design, Implementation and interest for the [Computer] Scientific Community, First OSCAR Symposium, Sherbrooke May 1114, Canada, 2003.

2. Zhang, W. (April, 2004) "Linux Virtual Server for Scalable Network Services", Available online: http://www.linuxvirtualserver.org/docs/scheduling.html.

3. Dantas, M.A.R. and Zaluska, E.J., Efficient Scheduling of MPI Applications on Networks of Workstations, FGCS Journal, V 13, pp. 489-499, 1998.

4. Casavant T.H. and Khul J.G., "A Taxonomy of Scheduling in General-Purpose Distributed Computing Systems", IEEE Trans. On Software Eng., vol. 14, no. 2, pp. 141-154, Feb. 1988.

5. Zhou S., "A Trace-driven Simulation Study of Dynamic Load Balancing", IEEE Trans. On Software Eng., vol. 14, no. 9, pp. 1327-1341, sep. 1988.

6. Dantas, M.A.R., Queiroz W.J. and Pfitscher G.H., "An Efficient Threshold Approach on Distributed Workstation Clusters", in HPC in Simulation, pp. 313-317, Washington, USA, 2000.

7. Papadimitriou, C. and Steilglitz, K., Combinatorial Optimization: Algoritms and Complexity, Dover Publications, 1998.

8. Goldberg, D.E., "Genetic Algorithm in Search, Optimization, and Machine Learning", Addison-Wesley, 1989.

9. Powell, M.L. and Miller, B.P., "Process migration in DEMOS/MP", in Proc. 9th ACM Symp. Operat. Syst. Principles, 1983, pp.110-119. 